\title{
Audit of Home Parenteral Nutrition in Palliative Care Patients
}

Clare Shaw ${ }^{1,2,}$, Lucy Eldridge ${ }^{1,}$ Joanne Droney ${ }^{3}$, Angela Halley ${ }^{3}$

1. Department of Nutrition and Dietetics, The Royal Marsden NHS Foundation Trust; 2. RM/ICR Biomedical Research Centre 3. Palliative Care and Symptom Control Team, The Royal Marsden NHS Foundation Trust, London and Sutton;

\section{Introduction}

The provision of parenteral nutrition in palliative care is controversial in the United Kingdom. Home Parenteral Nutrition (HPN) is not recommended for patients with incurable disease and a short life expectancy. European guidance states that HPN may be considered for patients with malignant obstruction, a prognosis of more than 3 months and a WHO performance status of less than $2^{\dagger}$.

\section{Objectives}

To determine whether the provision of home parenteral nutrition for palliative patients at a tertiary cancer centre in the UK was in accordance with European guidance.

\section{Method}

A retrospective audit of patient records was undertaken for patients who received HPN between June 2012 to May 2017

\section{Results}

34 patients were considered for home parenteral nutrition and 22 patients were discharged from The Royal Marsden Hospital for HPN.

Arrangements for setting up HPN were made in conjunction with an Intestinal Failure (IF) unit closest to the patient's home or via remote set up under the guidance of the IF unit.

Baseline age of HPN patients were 56.5 years (range $31-84$ years)

The majority, 50\% were undergoing palliative chemotherapy with other treatment options being surgery (23\%), symptom control (23\%) and post stem cell transplant (5\%)

Figure 1: Diagnosis of HPN patients

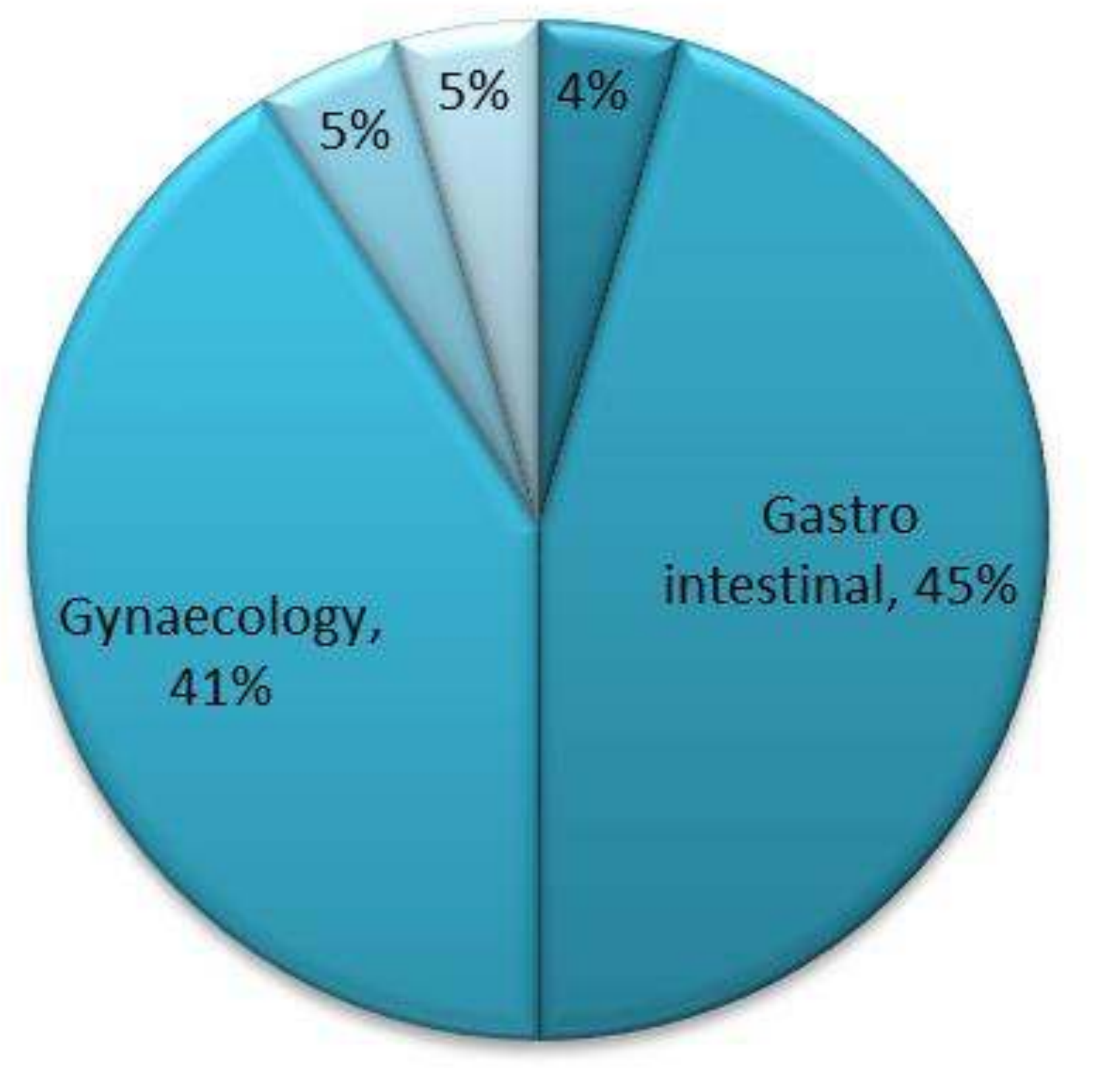

All patients referred for home PN were deemed to be appropriate in that they were not considered suitable for oral or enteral nutrition.

16 (73\%) of patients did not have a prognosis recorded during the HPN referral process

10 (45\%) of patients had a performance status recorded

6 (27\%) had a WHO performance status appropriate for HPN $(<2)$

13 (59\%) of patients were seen by the Palliative Care and Symptom control team.

Involvement of the Palliative Care and Symptom control team and a remote set up in liaison with the IF unit facilitated a reduced hospital stay.

Table 1: Length of hospital stay

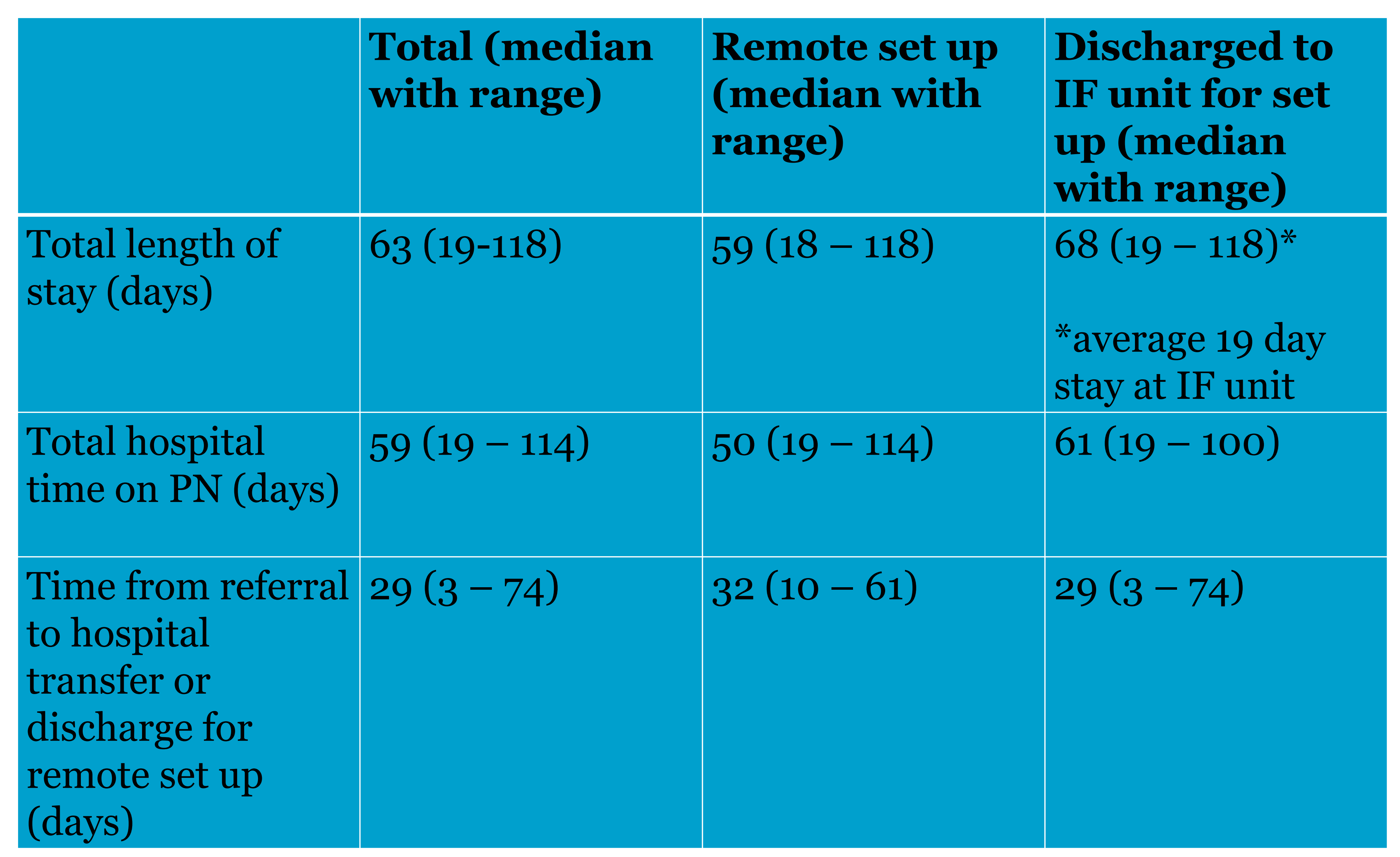

\section{Conclusions}

Although all patients were suitable for HPN there was poor documentation on prognosis and performance status. This may result in poor patient selection for the provision of HPN. Actions taken as a result of this audit have included

$>$ Dietitians have been taught to assess and record WHO and Karnofsky performance status

$>$ Development of a standard proforma for all patients being considered for HPN

$>$ Development of multi-disciplinary discussion of patients being considered for HPN

$>$ Development of video meetings with IF unit to discuss patient referral 